\title{
Efficacy of the controlled release of concentrated platelet lysate from a collagen/gelatin scaffold for dermis-like tissue regeneration.
}

\section{$\operatorname{AUTHOR}(\mathrm{S}):$}

Ito, Ran; Morimoto, Naoki; Pham, Liem Hieu; Taira, Tsuguyoshi; Kawai, Katsuya; Suzuki, Shigehiko

\section{CITATION:}

Ito, Ran ...[et al]. Efficacy of the controlled release of concentrated platelet lysate from a collagen/gelatin scaffold for dermis-like tissue regeneration.. Tissue engineering. Part A 2013, 19(11-12): 1398-1405

\section{ISSUE DATE:}

2013-04

\section{URL:}

http://hdl.handle.net/2433/174099

\section{RIGHT:}

(c) 2012 copyright Mary Ann Liebert, Inc.; "Tissue Engineering Part A" is available online at: http://online.liebertpub.com. 


\title{
Efficacy of the Controlled Release of Concentrated Platelet Lysate from a Collagen/Gelatin Scaffold for Dermis-Like Tissue Regeneration
}

\author{
Ran Ito, MD, ${ }^{1}$ Naoki Morimoto, MD, PhD, ${ }^{2}$ Liem Hieu Pham, MD, ${ }^{1}$ Tsuguyoshi Taira, MAg, ${ }^{3}$ \\ Katsuya Kawai, MD, PhD, and Shigehiko Suzuki, MD, PhD ${ }^{1}$
}

Introduction: A collagen/gelatin scaffold (CGS) can provide a sustained release of basic fibroblast growth factor (bFGF), which promotes wound healing. However, bFGF is approved for clinical use in Japan and China only. One potential alternative to bFGF is platelet lysate (PL), a safe and easily attainable source of a wide range of growth factors necessary for tissue repair. In the present study, we investigated the use of PL with CGS to repair wounds and identified the optimal concentration of PL for wound healing.

Materials and Methods: We generated PL from concentrated platelets harvested from individual healthy donors. We measured growth factors in PL. Transforming growth factor (TGF)- $\beta 1$, platelet-derived growth factor (PDGF)-BB, vascular endothelial growth factor (VEGF), and bFGF were selected because they were the major growth factors contained in platelets and showed the greatest ability to enhance the maturation of newly formed blood vessels. Pieces of CGS impregnated with PL solution $(\times 1, \times 2, \times 3$, or $\times 4$ concentrated $)$ or normal saline solution (NSS) were implanted into full-thickness skin defects on the backs of mice. We evaluated the wound area, neoepithelium length, and total area of newly formed capillaries in the implanted CGS.

Results: Our release experiments revealed that PDGF-BB and TGF- $\beta 1$ were released from CGS incubated with collagenase in a sustained manner. CGS impregnated with concentrated PL was more effective than CGS impregnated with NSS in all evaluated items. The $\times 2$ concentrated PL accelerated wound healing and enhanced cell proliferation and vessel growth in granulation tissue.

Conclusion: Our findings indicate that CGS can bind to TGF- $\beta 1$ and PDGF-BB and release these growth factors in a sustained manner. $\times 2$ concentrated PL-impregnated CGS accelerates the formation of dermis-like tissue.

\section{Introduction}

$\mathbf{U}$ SING A MODIFYING VERSION of the technique proposed by Yannas and Burke, ${ }^{1-3}$ we developed a novel collagen/ gelatin scaffold (CGS) for open wound repair that can provide a sustained release of basic fibroblast growth factor (bFGF), which promotes neoepithelium growth. The CGS we developed contains a mass percentage of $10 \%$ negatively charged concentration of acidic gelatin that is able to bind to positively charged growth factors such as bFGF and later release these growth factors in a sustained manner. In a previous study, we found that the biodegradation of CGS caused the scaffold to release bFGF and that bFGFimpregnated CGS implanted in full-thickness skin defects accelerated the formation dermal tissue. ${ }^{4,5}$ However, bFGF is currently approved for clinical use in Japan and China only.
One alternative to bFGF is platelet lysate (PL), which can be generated from common platelet units using a simple freeze-thaw procedure. PL can provide a substitute for cell culture supplement ${ }^{6,7}$ and is a safe and easily attainable source of a wide range of the growth factors that interact with cells during the wound-healing cascade and support the diverse processes that promote tissue revascularization and repair. Platelet aggregation initiates coagulation in bleeding vessels and releases growth factors that regulate wound healing events, 8,9 and depending on the complexity of the wound, platelets can employ the body's inherent capacities to boost healing and regenerative processes. ${ }^{10} \mathrm{PL}$ has been used clinically in a wide variety of surgical treatments for soft and hard tissue defects, ${ }^{11}$ including intractable wounds, ${ }^{12}$ calvaria, ${ }^{7}$ maxillofacial bone defects, ${ }^{6}$ cosmetic surgery, ${ }^{13,14}$ spinal problems, ${ }^{15}$ and neovascularization. ${ }^{16}$ However, the clinical application of PL is limited because of its short half-life

\footnotetext{
${ }^{1}$ Department of Plastic and Reconstructive Surgery, Graduate School of Medicine, Kyoto University, Kyoto, Japan.

${ }^{2}$ Department of Plastic and Reconstructive Surgery, Kansai Medical University, Osaka, Japan.

${ }^{3}$ Gunze Research and Development Center, Ayabe, Japan.
} 
and the variability of growth factor profiles among different donors. ${ }^{11,17,18}$ Because PL and the growth factors dissolve into blood stream within days after their injection, it is difficult to maintain their presence in the tissue long enough to promote effective tissue regeneration.

The purpose of the present study was to investigate the efficacy of PL-impregnated CGS in promoting dermis-like tissue formation. To conduct this investigation, we used a freeze-dry procedure to generate concentrated PL that would offset the variability in growth factor profiles as well as a controlled-release system utilizing biodegradable CGS that facilitates concentrated PL's sustained release of major growth factors to maintain their effect.

\section{Materials and Methods}

\section{Animals}

Eight-week-old C57BL/6JJcl mice (CLEA Japan, Inc., Osaka Japan) were maintained in the Institute of Laboratory Animals, Graduate School of Medicine, Kyoto University, in compliance with protocols established by the university's Animal Research Committee.

\section{Preparation of CGS}

We used gelatin isolated from pig dermis with an isoelectric point (IEP) of 5.0 and molecular weight of 99,000 (Nippi, Osaka, Japan) and atelocollagen isolated from pig tendons with an IEP of 8.5 and molecular weight of 300,000 (Nitta Gelatin, Osaka, Japan) to create the CGS. We prepared CGS as described previously. ${ }^{1-3}$ Briefly, CGS with gelatin concentrations of a mass percentage of $10 \%$ of the total solute was prepared by mixing a mass percentage of $3 \%$ gelatin solution into a mass percentage of $0.3 \%$ collagen solution. The foaming solution was poured into a mold, frozen rapidly, and freeze-dried. The resulting collagen-gelatin sheet was stabilized by immersing it in a crosslinking agent $\left(0.2 \%\right.$ glutaraldehyde) for $24 \mathrm{~h}$ at $4^{\circ} \mathrm{C}$ and then freeze-drying it. We used silicone solution (Shin-Etsu Chemical, Tokyo, Japan) to make $200-\mu$ m-thick silicone sheets that would be used to protect the implanted CGS.

\section{Generation of human supplements}

PLs were generated from concentrated platelets that had been harvested from 6 healthy humans. The concentrated platelets were frozen at $-80^{\circ} \mathrm{C}$ and thawed at $37^{\circ} \mathrm{C}$ twice to lysate the platelets for release of growth factors. To remove the membrane fragments, we centrifuged the lysates at 3000 $g$ for $30 \mathrm{~min}$ and filtered the supernatant through a $0.2-\mu \mathrm{m}$ Steradisc (Kurabo, Osaka, Japan). To prevent gelatinization, we added $2 \mathrm{U} / \mathrm{mL}$ heparin to the media, which was then stored at $-80^{\circ} \mathrm{C}$ until use.

Concentrated PLs were prepared using a freeze-dryer (VD250R; TAITEC Co., Saitama, Japan) according to the manufacturer's instructions and stored at $4^{\circ} \mathrm{C}$ until use. To create a $\times 4$ concentration of PLs, we reconstituted freeze-dried PLs with an amount of normal saline solution (NSS; Otsuka Pharmaceutical, Tokyo, Japan) that was one-fourth the original volume of the PL media. To create a $\times 3$ concentration of PLs, we added NSS to the $\times 4$ concentration to produce a $4 / 3$ fold dilution of the $\times 4$ concentration. To create a $\times 2$ concentration of PLs, we added NSS to the $\times 4$ concentration to produce a two-fold dilution of $\times 4$ concentration.

\section{Measurement of growth factors in PL}

The platelets contained in $\alpha$-granules release the following factors: angiopoietin-2, calcium, coagulation factors, epithelial cell growth factor, epidermal growth factor, ${ }^{19-24}$ fibronectin, fibrinogen, bFGF of FGF2, fibronectin, histamine, insulin-like growth factor- $1,{ }^{21}$ interleukin-1, platelet-derived angiogenesis factor, ${ }^{19,22,24,25}$ platelet-derived endothelial cell growth factor, ${ }^{19}$ PDGF (AA, BB, and AB isomers), ${ }^{11,19-22,26,27}$ platelet factor $4,22,24,25$ serotonin, TGF- $\beta,^{11,13,19-22,24-27}$ TSP$1,{ }^{24}$ VEGF, ${ }^{11,24,26}$ and von Willebrand factor. ${ }^{28}$ Based on the profile of the growth factors released by the platelets that are involved in wound healing and processes that culminate in parenchymal cell proliferation and tissue regeneration, we selected TGF- $\beta 1$, PDGF-BB, VEGF, and bFGF as representative platelet growth factors because they were produced in the largest amounts, $7,29,30$ and showed greatest ability to enhance the maturation of newly formed blood vessels. ${ }^{16,31-34}$ To quantify the amounts of the growth factors in the PLs, we used enzyme-linked immunosorbent assay (ELISA) and transforming growth factor (TGF)- $\beta 1$, platelet-derived growth factor (PDGF)-BB, vascular endothelial growth factor (VEGF), and bFGF kits (R\&D Systems, Minneapolis, MN) according to the manufacturer's instructions. Samples of PL from six humans were added to 96-well plates coated with anti-growth factor antibodies. After incubating the plates and removing the unbound substances, we added an enzyme-coupled secondary antibody to the well plates. After the color reactions occurred, we used a spectrophotometer to measure the optical densities of each product at the appropriate wavelength.

\section{Release test of PL from CGS}

To evaluate the release of PL from CGS, pieces of CGS $(1.0 \times 1.5 \times 0.3 \mathrm{~cm})$ were added to $250 \mu \mathrm{L}$ of phosphate-buffered saline (PBS; Invitrogen, Tokyo, Japan) and stored in a $\mathrm{CO}_{2}$ incubator at $37^{\circ} \mathrm{C}$. For $24 \mathrm{~h}$, we removed $200 \mu \mathrm{L}$ of the PBS release media for sampling at different times and replaced the media with $200 \mu \mathrm{L}$ of fresh PBS. At $24 \mathrm{~h}$, we removed $200 \mu \mathrm{L}$ of the PBS release media for sampling and replaced it with $200 \mu \mathrm{L}$ of $2 \mathrm{U} / \mathrm{mL}$ collagenase type A1 (Sigma Chemical, St. Louis, MO). We continued to remove $200 \mu \mathrm{L}$ of the PBS release media and replace it with $200 \mu \mathrm{L}$ of $2 \mathrm{U} / \mathrm{mL}$ collagenase type A1 at different times for the next $24 \mathrm{~h}$. The experiment was performed independently for three samples and three controls (NSS) at every sampling time.

To quantify the amounts of TGF- $\beta 1$ and PDGF-BB in the samples, we used ELISA and TGF- $\beta 1$ and PDGF-BB ELISA kits (R\&D Systems, Minneapolis, MN) according to the manufacturer's instructions. The samples $(n=3)$ and controls (NSS, $n=3$ ) were added to 96-well plates coated with antigrowth factor antibodies. After incubating the plates and removing the unbound substances, we added an enzymecoupled secondary antibody to the well plates. After the color reactions occurred, we used a spectrophotometer to measure the optical densities of each product at the appropriate wavelength.

\section{Impregnation of CGS with PL}

Pieces of CGS (8-mm diameter, $1.5 \mathrm{~mm}$ thick) were placed in $10-\mathrm{cm}$ tissue culture dishes (Falcon; Falcon, NY). We 
applied $50 \mu \mathrm{L}$ of NSS (control), PL, or $\times 2, \times 3$, or $\times 4$ concentrated PL to the CGS and then incubated the impregnated CGS overnight at $4^{\circ} \mathrm{C}$.

\section{Implantation of CGS}

To determine the concentration of PL in CGS that is most effective in promoting wound healing, we first implanted pieces of CGS impregnated with different concentrations of PL into full-thickness skin defects on the backs of mice. Eight-week-old C57BL/6JJcl mice were anesthetized by intraperitoneal injection of $26.3 \mathrm{mg}$ pentobarbital (Abbott Laboratories, North Chicago, IL). After depilating the mice, we used an 8-mm-diameter skin punch biopsy tool (Kai Industries, Gifu, Japan) to create a full-thickness skin defect (including the panniculus carnosus) on the back of each mouse. After dividing the mice into five groups of six mice each, we implanted pieces of CGS impregnated with NSS (control), PL, or $\times 2, \times 3$, or $\times 4$ concentrated PL into the defects and sutured the CGS to the margins of the skin wound with 5-0 nylon sutures (Johnson \& Johnson, Tokyo, Japan). The silicone sheets were then placed over the defects to keep the inner sponge layer wet and prevent bacterial infection.

\section{Assessment of the wound area and histologic assessment of neoepithelization}

Mice were killed by carbon dioxide inhalation 1, 2, or 3 weeks after CGS implantation. The silicone sheets were removed, and each treated wound area, which was expressed as a percentage of the area of the original 8- $\mathrm{mm}$ wound, was photographed and measured using ImageJ software (version 1.38; Wayne Rasband, NIH, Maryland). We used a scalpel and scissors to harvest the implanted CGS. The CGS specimens were fixed with $20 \%$ formalin, embedded in paraffin, cut axially into $4-\mu \mathrm{m}$-thick sections, and then stained with hematoxylin and eosin. We then used a light microscope to measure the length of each specimen's neoepithelium from the outermost hair root of the marginal skin on each side of each cross section.

\section{Immunohistological staining of newly formed capillaries and evaluation of capillary area}

To detect newly formed capillaries in the implanted CGS, we performed immunohistological staining of von Willebrand factor. After the harvested CGS sections had been deparaffinized and rehydrated, they were incubated in PBS with $0.1 \%$ trypsin (Vector Laboratories, Inc., Burlingame, CA) for $15 \mathrm{~min}$ at $37^{\circ} \mathrm{C}$. Anti-von Willebrand factor rabbit polyclonal antibody (Dako Japan, Tokyo, Japan) was used as the primary antibody (1:250 dilution), and En Vision + Rabbit/HRP (Dako Japan) was used as the secondary antibody. The sections were exposed to 3-3'-diaminobenzidine$4 \mathrm{HCl}$ (Dako Japan) for $2 \mathrm{~min}$ at room temperature and then counterstained with hematoxylin.

Digital light micrographs of sections beyond the muscle layers were taken at $100 \times$ magnification. The cross-sectioned area of stained capillaries beyond the muscle layer in each wound was measured using the NIS-Elements D imaging software program (version 2. 20; Nikon, Tokyo, Japan). Measurements were performed in the central region of a 500$\mu \mathrm{m}$ diameter in each section.

\section{Statistical analysis}

We used an analysis of variance and a Fisher protected least significant difference test to compare differences between groups. All data are expressed as means \pm standard errors. $p$ values $<0.05$ were considered statistically significant. The Microsoft Excel software program with the Statcel software add-in (Oms publishing, Inc., Tokyo, Japan) was used for all statistical analyses.

\section{Results \\ Growth factors in PL}

The concentrations of the principal growth factors released from PL are shown in Table 1. Various growth factors were released from PL after the freeze-thaw activation. The amounts of growth factors in concentrated PL are shown in Figure 1.

\section{$P L$ release from $C G S$}

Our growth factor release experiments revealed that CGS could bind to and release TGF- $\beta 1$ and PDGF-BB in vivo and that this release depended on the biodegradation of collagenase (Fig. 2). During the initial 24-h incubation period, CGS incubated with PBS without collagenase did not release TGF- $\beta 1$ or PDGF-BB in a sustained manner but did release initial bursts of small amounts of the growth factors. In contrast, during the second 24-h incubation period, CGS incubated with collagenase-containing PBS released TGF- $\beta 1$ and PDGF-BB in a sustained manner. During the first 24-h period, the CGS incubated with PBS without collagenase released up to $50 \%$ of the growth factors; during the second 24-h period, collagenase degradation caused the CGS to release the remaining $50 \%$ of the growth factors. A similar addition effect of collagenase occurred on the time profile of CGS degradation. The time profiles of TGF- $\beta 1$, PDGF-BB, and CGS degradations had good correlation.

\section{Wound area}

The gross appearances of wounds 2 weeks after the implantation of CGS are shown in Figure 3A. Three weeks after CGS implantation, the wounds treated with PL-impregnated CGS had almost completely epithelized. The percentage of original wound area remaining over time is shown in Figure 3B. One, two, and three weeks after CGS implantation, the areas of the wounds in the mice treated with PL-impregnated

Table 1. Concentration of Growth Factors in Platelet Lysate

\begin{tabular}{lc}
\hline Growth factor & Concentration $(\mathrm{pg} / \mathrm{mL})$ \\
\hline TGF- $\beta 1$ & $103449 \pm 7137$ \\
PDGF-BB & $5112 \pm 1200$ \\
VEGF & $1568 \pm 521$ \\
bFGF & $75.1 \pm 11.1$
\end{tabular}

The concentrations of TGF- $\beta 1$, PDGF-BB, VEGF, and bFGF released from PL. Samples of PL $(n=6)$ were measured using enzyme-linked immunosorbent assay.

TGF, transforming growth factor; PDGF, platelet-derived growth factor; VEGF, vascular endothelial growth factor; bFGF, basic fibroblast growth factor; PL, platelet lysate. 

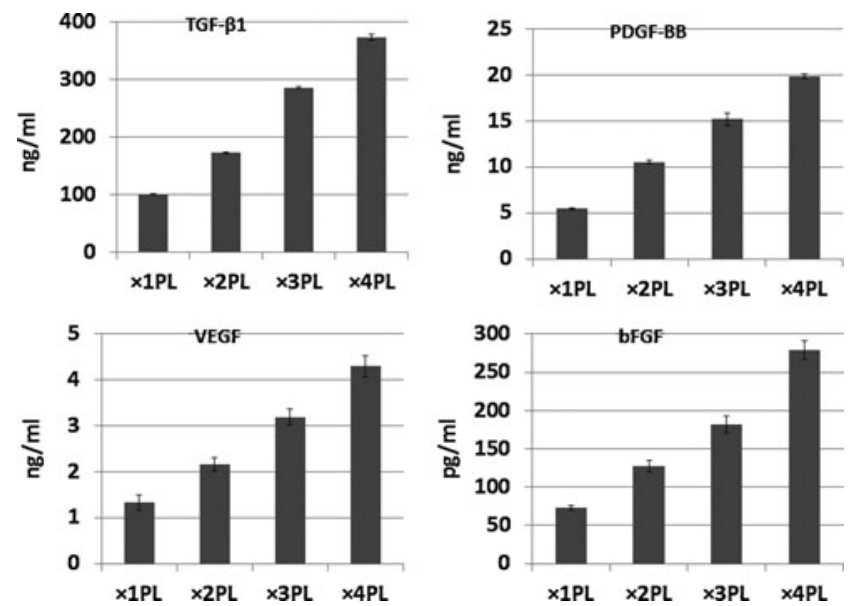

FIG. 1. The mean amounts of growth factors \pm standard deviations in different concentrations of platelet lysate (PL). Samples of PL $(n=6)$ were measured using enzyme-linked immunosorbent assay.

CGS were significantly smaller than those in the control group. Two weeks after CGS implantation, the areas of the wounds treated with CGS impregnated with $\times 2$ concentrated PL were significantly smaller than those of the wounds treated with CGS impregnated with $\times 3$ or $\times 4$ concentrated PL.

\section{Histologic assessment of neoepithelium length}

We observed newly formed epithelia in wounds treated with PL-impregnated CGS 2 weeks after CGS implantation (Fig. 4A). One and two weeks after CGS implantation, the

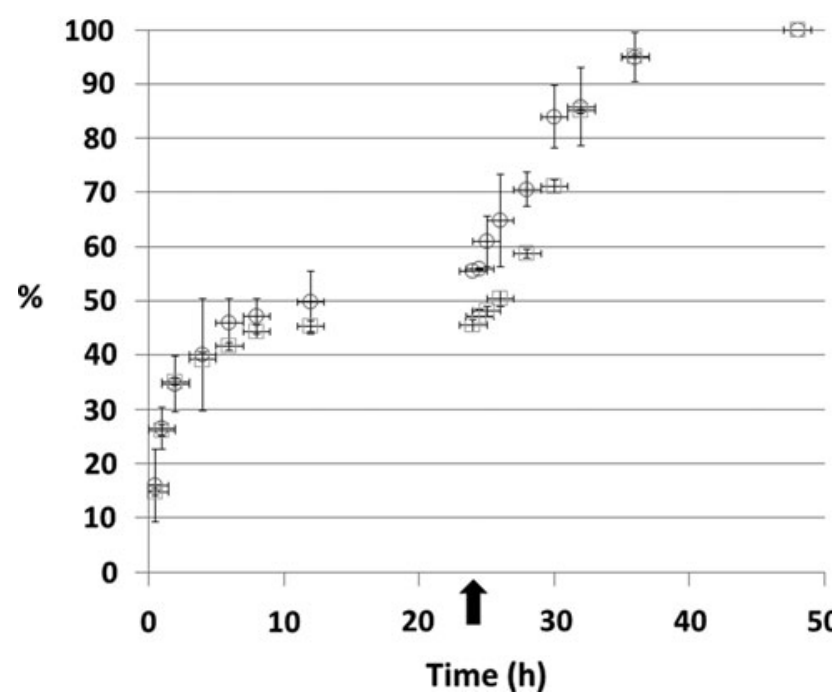

FIG. 2. The time course of the mean percentages of transforming growth factor (TGF)- $\beta 1$ and platelet-derived growth factor (PDGF)-BB release \pm standard deviations from CGS treated with $\times 2$ concentrated PL. The arrow indicates the time at which the addition of PBS solution was stopped and the addition of collagenase begun. The experiment was performed independently for three samples and three controls (NSS) at each sampling time. $\bigcirc$; TGF- $\beta 1, \square$; PDGF-BB

lengths of neoepithelium in wounds treated with CGS impregnated with $\times 2$ concentrated PL were significantly longer than those in the control group (Fig. 4B).

\section{Evaluation of newly formed capillaries in wounds}

CGS that had been immunohistologically stained for von Willebrand factor is shown in Figure 5A. Matrices of CGS were present in wounds treated with NSS-impregnated CGS but not those treated with PL-impregnated CGS. The gross area of the capillaries in the group treated with CGS impregnated with $\times 2$ concentrated PL was significantly larger than those in the control group and groups treated with CGS impregnated with $\times 3$ or $\times 4$ concentrated PL (Fig. 5B).

\section{Discussion}

In the present study, we found that CGS can bind to TGF$\beta 1$ and PDGF-BB and release these growth factors in a

A

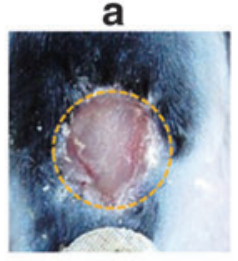

d

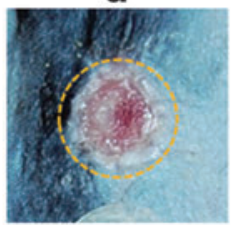

b

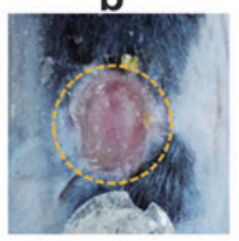

e

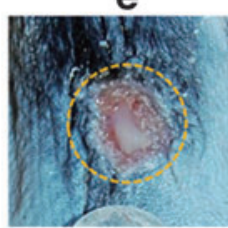

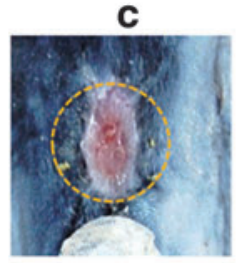

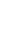


A

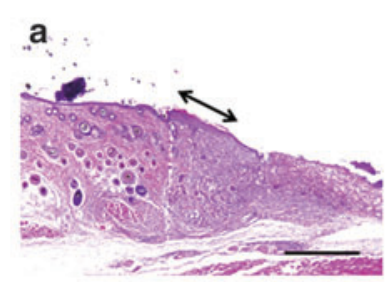

b

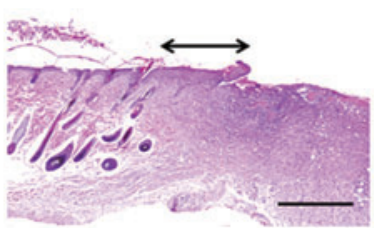

C

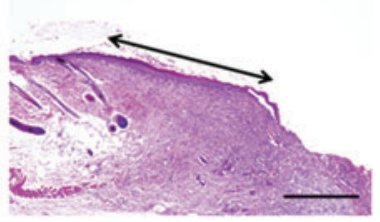

d

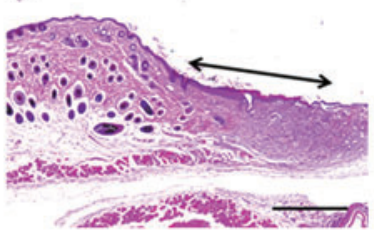

e
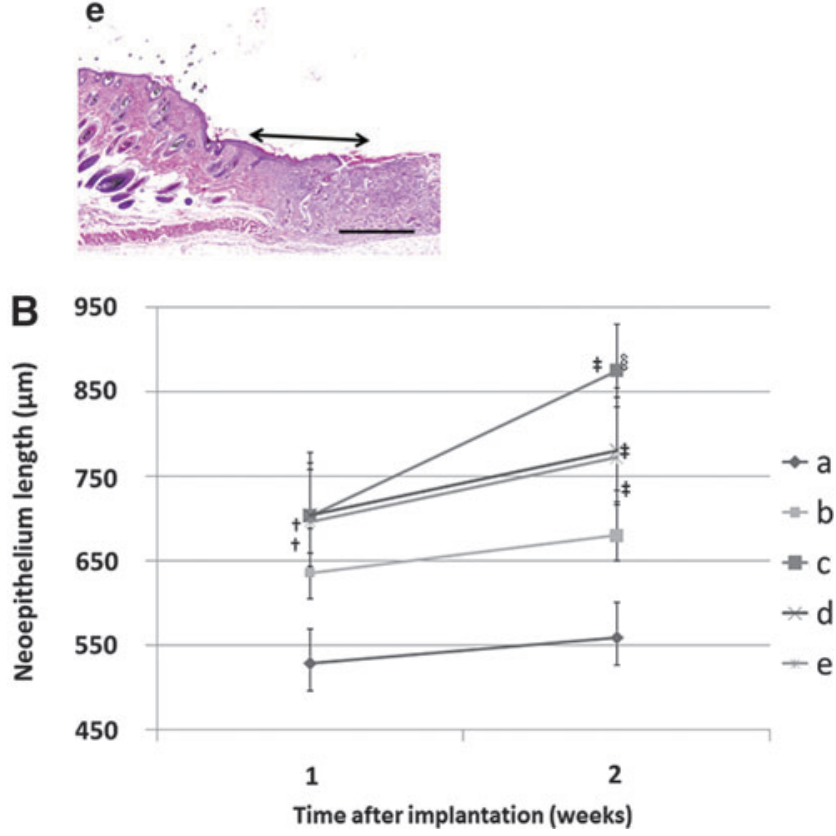

FIG. 4. (A) Hematoxylin and eosin staining micrographs of wounds treated with NSS (a), $\times 1$ concentrated PL (b), $\times 2$ concentrated PL (c), $\times 3$ concentrated PL (d), or $\times 4$ concentrated PL (e) 2 weeks after CGS implantation. Scale bar: $100 \mu \mathrm{m}$. The black arrows indicate the neoepithelium. (B) The time course of the change in the mean neoepithelium lengths $(\mu \mathrm{m}) \pm$ standard deviations of groups of six mice treated with NSS (a), $\times 1$ concentrated PL (b), $\times 2$ concentrated PL (c), $\times 3$ concentrated PL (d), or $\times 4$ concentrated PL (e) 2 weeks after CGS implantation. ${ }^{\dagger} p<0.05$ versus NSS; ${ }^{\S} p<0.01$ versus NSS, $\times 1$ concentrated PL; ${ }^{\star} p<0.01$ versus NSS. Color images available online at www.liebertpub.com/tea

sustained manner. Both growth factors were released according to the degradation of CGS, even when concentrated PL was applied. These results suggest that the release of multiple growth factors in concentrated PL from CGS helps heal wounds.

Although freeze-dried platelets have been reported to be effective for tissue regeneration, ${ }^{26,35}$ no study has investigated the efficacy of concentrated PL for wound healing. In living tissue, various growth factors interact with components of the extracellular matrix, such as acidic polysaccharides and collagen, through various intermolecular forces. ${ }^{36}$
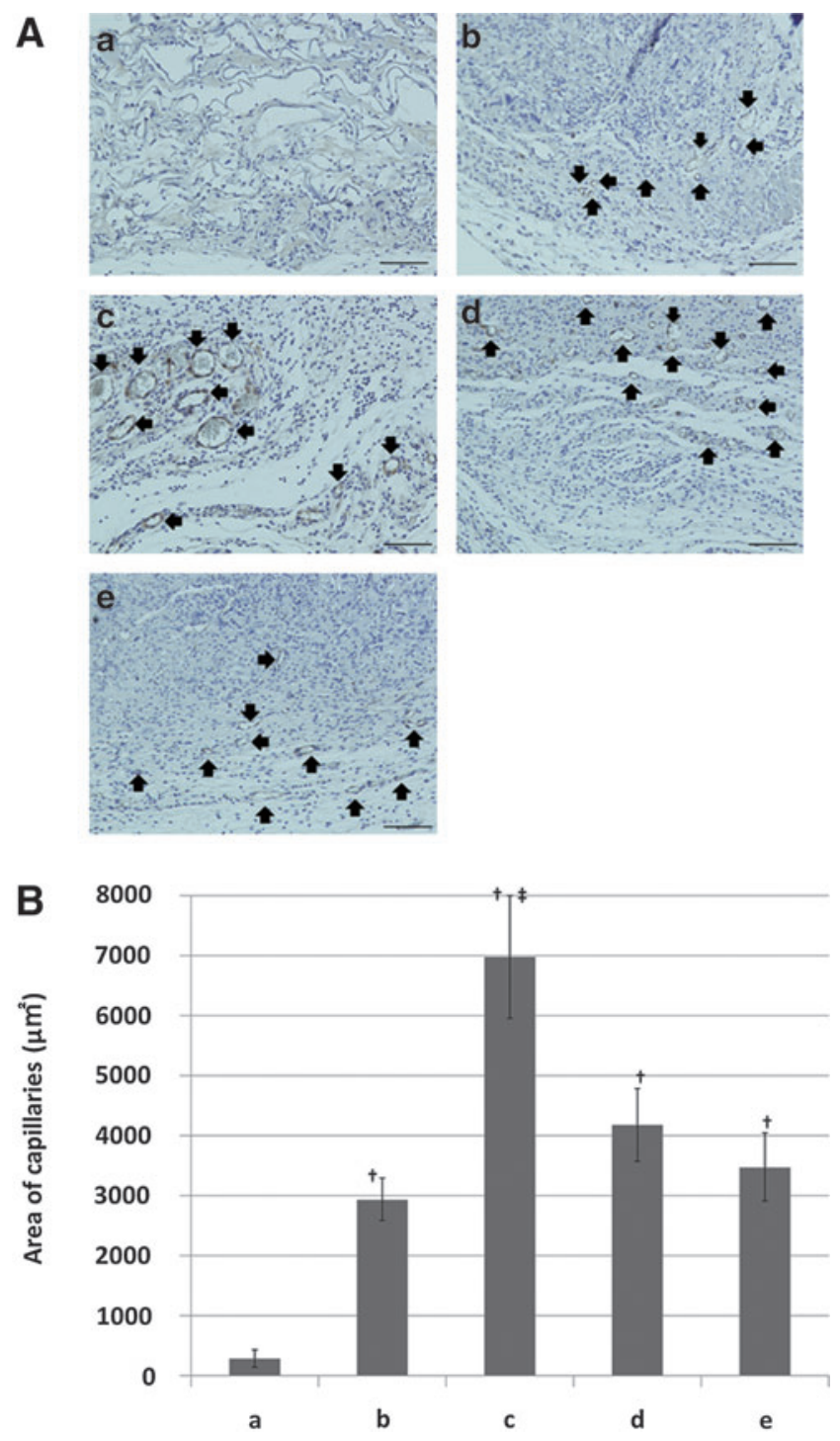

FIG. 5. (A) Immunohistological staining of the newly formed capillaries beyond the muscle layer in wounds treated with NSS $(\mathbf{a}), \times 1$ concentrated PL $(\mathbf{b}), \times 2$ concentrated PL (c), $\times 3$ concentrated PL (d), or $\times 4$ concentrated PL (e). The black arrows indicate newly formed capillaries. Scale bar: $100 \mu \mathrm{m}$. (B) The mean gross areas of capillaries \pm standard deviations of groups of six mice treated with CGS impregnated with NSS (a), $\times 1$ concentrated PL (b), $\times 2$ concentrated PL (c), $\times 3$ concentrated PL (d), or $\times 4$ concentrated PL (e). Scale bar: $100 \mu \mathrm{m} .{ }^{\dagger} p<0.01$ versus NSS; ${ }^{\star} p<0.01$ versus $\times 1 \mathrm{PL}, \times 3 \mathrm{PL}, \times 4 \mathrm{PL}$. Color images available online at www.liebertpub.com/tea

On the basis of this natural physicochemical interaction, we developed biodegradable CGS impregnated with bFGF to be used for full-thickness skin or mucosal defects. ${ }^{4,5,37}$

CGS can release concentrated PL in a sustained manner and facilitate the release of growth factors, which results in better angiogenesis and dermis-like tissue formation. In the present study, we found that $\times 2$ concentrated PL effectively accelerated wound healing, enhancing the proliferation of cell and vessel growth in granulation tissue without obvious inflammatory reactions. Moreover, the use of $\times 2$ concentrated PL 
elicited results that were not significantly different from those of our previous study, in which we used bFGF. ${ }^{5}$

In the present study, our finding that high concentrations of PL have not accelerated wound healing is consistent with those of previous studies, which showed that very high concentrations of PL have an inhibitory effect on the proliferation and viability of different cell types. ${ }^{38,39}$ For example, one study found that PL significantly enhanced the wound closure of keratinocyte-based tissue in a dose-dependent manner; however, this healing effect was retarded at concentrations 2.5 times the physiological level and higher. ${ }^{40}$ Furthermore, in wounded cell monolayers, optimal concentrations of PL have been found to induce a rapid actin cytoskeletal reorganization in agreement with the cells assuming an active cell migratory phenotype. $^{41,42}$

Our findings regarding PL were not dissimilar from those of earlier studies of bFGF. For example, bFGF treatment has been reported to elicit a bell-shaped dose response ${ }^{5,43-45}$; an excess amount of bFGF over the optimal range decreased the proliferation of keratinocytes ${ }^{43}$ and prolonged wound closure in vivo. ${ }^{4,45}$ Similarly, the results of the present study indicated that PL elicited a bell-shaped dose-response pattern with a peak at $\times 2$ concentrated PL, and the excess amount of platelet growth factors such as TGF- $\beta$ and PDGF$\mathrm{BB}$ over the optimal range reduced cell proliferation and prolonged wound closure in a manner similar to that of bFGF. The reduction in cell proliferation may have been due to the PL's release of thrombospondin (TSP)-1, which exists in significant quantities in PL and has been reported to be an important angiogenesis inhibitor. High concentrations of TSP-1 have been found to markedly inhibit cell proliferation. $^{39}$ Therefore, platelets' abundant secretion of TSP-1 might contribute to the antiproliferative effect via cell apoptosis. ${ }^{39}$ Moreover, several growth factors become intricately involved in angiogenesis. PDGF-BB has a potent arteriogenic effect that promotes differentiation of endothelial cells, ${ }^{46}$ VEGF is known to stimulate angiogenesis after ischemia, ${ }^{47}$ and TGF- $\beta$ promotes cell mitosis. ${ }^{31}$ However, other studies indicated that several growth factors, such as PDGF-BB and TGF- $\beta$, inhibit the angiogenic effect of bFGF. ${ }^{48-51}$ More studies are needed to determine what is responsible for the antiproliferative effect of high concentrations of PL.

On the other hand, different growth factors act in synergy to enhance tissue regeneration. ${ }^{52-54}$ For example, Hao et al. reported that the release of both VEGF and PDGF enhanced the maturation of newly formed blood vessels more than the release of only VEGF did. ${ }^{55}$ PL can release any drug capable of promoting the regeneration of various tissues. Thus, the findings of the present study could point the way to using platelets instead of bFGF in CGS for wound treatment. In addition, because CGS can simultaneously release agents in a sustained manner, the combination of concentrated PL- and bFGF-impregnated CGS could 1 day be used to further accelerate dermis-like tissue formation in wounds.

\section{Conclusion}

Our findings indicate that CGS can release these growth factors in a sustained manner. The $\times 2$ concentrated PLimpregnated CGS accelerates the formation of dermis-like tissue.

\section{Acknowledgment}

This work was supported by a Grant-in-Aid for Scientific Research from the Japan Society for the Promotion of Science.

\section{Disclosure Statement}

No competing financial interests exist.

\section{References}

1. Yannas, IV., and Burke, J.F. Design of an artificial skin. I. Basic design principles. J Biomed Mater Res 14, 65, 1980.

2. Suzuki, S., Matsuda, K., Isshiki, N., Tamada, Y., and Ikada, Y. Experimental study of a newly developed bilayer artificial skin. Biomaterials 11, 356, 1990.

3. Suzuki, S., Matsuda, K., Maruguchi, T., Nishimura, Y., and Ikada, Y. Further applications of "bilayer artificial skin". Br J Plast Surg 48, 222, 1995.

4. Takemoto, S., Morimoto, N., Kimura, Y., Taira, T., Kitagawa, T., Tomihata, K., et al. Preparation of collagen/gelatin sponge scaffold for sustained release of bFGF. Tissue Eng Part A 14, 1629, 2008.

5. Kanda, N., Morimoto, N., Takemoto, S., Ayvazyan, A.A., Kawai, K., Sakamoto, Y., et al. Efficacy of novel collagen/ gelatin scaffold with sustained release of basic fibroblast growth factor for dermis-like tissue regeneration. Ann Plast Surg 69, 569, 2012.

6. Marx, R.E., Carlson, E.R., Eichstaedt, R.M., Schimmele, S.R., Strauss, J.E., and Georgeff, K.R. Platelet-rich plasma: growth factor enhancement for bone grafts. Oral Surg Oral Med Oral Pathol Oral Radiol Endod 85, 638, 1998.

7. Hokugo, A., Sawada, Y., Hokugo, R., Iwamura, H., Kobuchi, M., Kambara, T., et al. Controlled release of platelet growth factors enhances bone regeneration at rabbit calvaria. Oral Surg Oral Med Oral Pathol Oral Radiol Endod 104, 44, 2007.

8. Martinowitz, U., and Spotnitz, W.D. Fibrin tissue adhesives. Thromb Haemost 78, 661, 1997.

9. Folkman, J., Browder, T., and Palmblad, J. Angiogenesis research: guidelines for translation to clinical application. Thromb Haemost 86, 23, 2001.

10. Chen, F.M., Zhang, M., and Wu, Z.F. Toward delivery of multiple growth factors in tissue engineering. Biomaterials 31, 6279, 2010.

11. Marx, R.E. Platelet-rich plasma: evidence to support its use. J Oral Maxillofac Surg 62, 489, 2004.

12. Margolis, D.J., Kantor, J., Santanna, J., Strom, B.L., and Berlin, J.A. Effectiveness of platelet releasate for the treatment of diabetic neuropathic foot ulcers. Diabetes Care 24, 483, 2001.

13. Bhanot, S., and Alex, J.C. Current applications of platelet gels in facial plastic surgery. Facial Plast Surg 18, 27, 2002.

14. Man, D., Plosker, H., and Winland-Brown, J.E. The use of autologous platelet-rich plasma (platelet gel) and autologous platelet-poor plasma (fibrin glue) in cosmetic surgery. Plast Reconstr Surg 107, 229, 2001.

15. Hee, H.T., Majd, M.E., Holt, R.T., and Myers, L. Do autologous growth factors enhance transforaminal lumbar interbody fusion? Eur Spine J, 12, 400, 2003.

16. Bir, S.C., Esaki, J., Marui, A., Yamahara, K., Tsubota, H., Ikeda, T., et al. Angiogenic properties of sustained release platelet-rich plasma: characterization in-vitro and in the ischemic hind limb of the mouse. J Vasc Surg 50, 870, 2009.

17. Pietramaggiori, G., Scherer, S.S., Mathews, J.C., Alperovich, M., Yang, H.J., Neuwalder, J., et al. Healing modulation 
induced by freeze-dried platelet-rich plasma and micronized allogenic dermis in a diabetic wound model. Wound Repair Regen 16, 218, 2008.

18. Sugimori, E., Shintani, S., Ishikawa, K., and Hamakawa, H. Effects of apatite foam combined with platelet-rich plasma on regeneration of bone defects. Dent Mater J 25, 591, 2006.

19. Steed, D.L., Goslen, J.B., Holloway, G.A., Malone, J.M., Bunt, T.J., and Webster, M.W. Randomized prospective doubleblind trial in healing chronic diabetic foot ulcers. CT-102 activated platelet supernatant, topical versus placebo. Diabetes Care 15, 1598, 1992.

20. Robson, M.C., Phillips, L.G., Thomason, A., Altrock, B.W., Pence, P.C., Heggers, J.P., et al. Recombinant human plateletderived growth factor-BB for the treatment of chronic pressure ulcers. Ann Plast Surg 29, 193, 1992.

21. Weibrich, G., Kleis, W.K., Kunz-Kostomanolakis, M., Loos, A.H., and Wagner, W. Correlation of platelet concentration in platelet-rich plasma to the extraction method, age, sex, and platelet count of the donor. Int J Oral Maxillofac Implants 16, 693, 2001.

22. Henderson, J.L., Cupp, C.L., Ross, E.V., Shick, P.C., Keefe, M.A., Wester, D.C., et al. The effects of autologous platelet gel on wound healing. Ear Nose Throat J 82, 598, 2003.

23. El-Sharkawy, H., Kantarci, A., Deady, J., Hasturk, H., Liu, H., Alshahat, M., et al. Platelet-rich plasma: growth factors and pro- and anti-inflammatory properties. J Periodontol 78, 661, 2007.

24. Nikolidakis, D., and Jansen, J.A. The biology of platelet-rich plasma and its application in oral surgery: literature review. Tissue Eng Part B Rev 14, 249, 2008.

25. Knighton, D.R., Doucette, M., Fiegel, V.D., Ciresi, K., Butler, E., and Austin, L. The use of platelet derived wound healing formula in human clinical trials. Prog Clin Biol Res 266, 319, 1988.

26. Pietramaggiori, G., Kaipainen, A., Czeczuga, J.M., Wagner, C.T., and Orgill, D.P. Freeze-dried platelet-rich plasma shows beneficial healing properties in chronic wounds. Wound Repair Regen 14, 573, 2006.

27. Gonshor, A. Technique for producing platelet-rich plasma and platelet concentrate: background and process. Int J Periodontics Restorative Dent 22, 547, 2002.

28. Harrison, P., and Cramer, E.M. Platelet alpha-granules. Blood Rev 7, 52, 1993.

29. Peattie, R.A., Pike, D.B., Yu, B., Cai, S., Shu, X.Z., Prestwich, G.D., et al. Effect of gelatin on heparin regulation of cytokine release from hyaluronan-based hydrogels. Drug Deliv 15, 389, 2008

30. Tabata, Y., Hong, L., Miyamoto, S., Miyao, M., Hashimoto, N., and Ikada, Y. Bone formation at a rabbit skull defect by autologous bone marrow cells combined with gelatin microspheres containing TGF-beta1. J Biomater Sci Polym Ed 11, 891, 2000.

31. Schultz, G.S., and Grant, M.B. Neovascular growth factors. Eye (Lond) 5, 170, 1991.

32. Nicosia, R.F., Nicosia, S.V., and Smith, M. Vascular endothelial growth factor, platelet-derived growth factor, and insulin-like growth factor-1 promote rat aortic angiogenesis in vitro. Am J Pathol 145, 1023, 1994.

33. Hellberg, C., Ostman, A., and Heldin, C.H. PDGF and vessel maturation. Recent Results Cancer Res 180, 103, 2010.

34. Bertrand-Duchesne, M.P., Grenier, D., and Gagnon, G. Epidermal growth factor released from platelet-rich plasma promotes endothelial cell proliferation in vitro. J Periodontal Res 45, 87, 2010.
35. Hoshi, R., Murata, S., Matsuo, R., Myronovych, A., Hashimoto, I., Ikeda, H., et al. Freeze-dried platelets promote hepatocyte proliferation in mice. Cryobiology 55, 255, 2007.

36. Taipale, J., and Keski-Oja, J. Growth factors in the extracellular matrix. FASEB J 11, 51, 1997.

37. Ayvazyan, A., Morimoto, N., Kanda, N., Takemoto, S., Kawai, K., Sakamoto, Y., et al. Collagen-gelatin scaffold impregnated with bFGF accelerates palatal wound healing of palatal mucosa in dogs. J Surg Res 171, e247, 2011.

38. Smith, S.E., and Roukis, T.S. Bone and wound healing augmentation with platelet-rich plasma. Clin Podiatr Med Surg 26, 559, 2009.

39. Hsu, C.W., Yuan, K., and Tseng, C.C. The negative effect of platelet-rich plasma on the growth of human cells is associated with secreted thrombospondin-1. Oral Surg Oral Med Oral Pathol Oral Radiol Endod 107, 185, 2009.

40. El Backly, R., Ulivi, V., Tonachini, L., Cancedda, R., Descalzi, F., and Mastrogiacoma, M. Platelet lysate induces in vitro wound healing of human keratinocytes associated with a strong proinflammatory response. Tissue Eng Part A 17, 1787, 2011.

41. Henry, G., Li, W., Garner, W., and Woodley, D.T. Migration of human keratinocytes in plasma and serum and wound reepithelialisation. Lancet 361, 574, 2003.

42. Ridley, A.J., Schwartz, M.A., Burridge, K., Firtel, R.A., Ginsberg, M.H., Borisy, G., et al. Cell migration: integrating signals from front to back. Science 302, 1704, 2003.

43. Tanaka, E., Ase, K., Okuda, T., Okumura, M., and Nogimori, K. Mechanism of acceleration of wound healing by basic fibroblast growth factor in genetically diabetic mice. Biol Pharm Bull 19, 1141, 1996.

44. Okumura, M., Okuda, T., Nakamura, T., and Yajima, M. Acceleration of wound healing in diabetic mice by basic fibroblast growth factor. Biol Pharm Bull 19, 530, 1996.

45. Motomura, K., Hagiwara, A., Komi-Kuramochi, A., Hanyu, Y., Honda, E., Suzuki, M., et al. An FGF1:FGF2 chimeric growth factor exhibits universal FGF receptor specificity, enhanced stability and augmented activity useful for epithelial proliferation and radioprotection. Biochim Biophys Acta 1780, 1432, 2008.

46. Carmeliet, P. Angiogenesis in health and disease. Nat Med 9, 653, 2003.

47. Ferrara, N. Role of vascular endothelial growth factor in regulation of physiological angiogenesis. Am J Physiol Cell Physiol 280, C1358, 2001.

48. Müller, G., Behrens, J., Nussbaumer, U., Böhlen, P., and Birchmeier, W. Inhibitory action of transforming growth factor beta on endothelial cells. Proc Natl Acad Sci U S A 84, 5600, 1987.

49. Facchiano, A., De Marchis, F., Turchetti, E., Facchiano, F., Guglielmi, M., Denaro, A., et al. The chemotactic and mitogenic effects of platelet-derived growth factor-BB on rat aorta smooth muscle cells are inhibited by basic fibroblast growth factor. J Cell Sci 113, 2855, 2000.

50. De Marchis, F., Ribatti, D., Giampietri, C., Lentini, A., Faraone, D., Scoccianti, M., et al. Platelet-derived growth factor inhibits basic fibroblast growth factor angiogenic properties in vitro and in vivo through its alpha receptor. Blood 99, 2045, 2002.

51. Tengood, J.E., Ridenour, R., Brodsky, R., Russell, A.J., and Little, S.R. Sequential delivery of basic fibroblast growth factor and platelet-derived growth factor for angiogenesis. Tissue Eng Part A 17, 1181, 2011.

52. Ratanavaraporn, J., Furuya, H., Kohara, H., and Tabata, Y. Synergistic effects of the dual release of stromal cell-derived 
factor- 1 and bone morphogenetic protein-2 from hydrogels on bone regeneration. Biomaterials 32, 2797, 2011.

53. Peng, H., Wright, V., Usas, A., Gearhart, B., Shen, H.C., Cummins, J., et al. Synergistic enhancement of bone formation and healing by stem cell-expressed VEGF and bone morphogenetic protein-4. J Clin Invest 110, 751, 2002.

54. Patel, Z.S., Young, S., Tabata, Y., Jansen, J.A., Wong, M.E., and Mikos, A.G. Dual delivery of an angiogenic and an osteogenic growth factor for bone regeneration in a critical size defect model. Bone 43, 931, 2008.

55. Hao, X., Silva, E.A., Månsson-Broberg, A., Grinnemo, K.H., Siddiqui, A.J., Dellgren, G., et al. Angiogenic effects of sequential release of VEGF-A165 and PDGF-BB with alginate hydrogels after myocardial infarction. Cardiovasc Res 75, 178, 2007.
Address correspondence to:

Ran Ito, $M D$

Department of Plastic and Reconstructive Surgery Graduate School of Medicine Kyoto University

54 Kawahara-cho, Syogoin

Sakyo-ku

Kyoto 606-8507

Japan

E-mail: ran_ito@kuhp.kyoto-u.ac.jp

Received: June 15, 2012

Accepted: January 16, 2013

Online Publication Date: March 26, 2013 